\title{
STUDY OF SKIN MICROBIOTA IMPAIRED BY SKIN HYGIENE HABITS
}

S. Leoty Okombi, P. Moussou, V. Andre-Frei and S. Pain

BASF Beauty Care Solutions France, 32 rue Saint Jean de Dieu, Lyon, France

\section{INTRODUCTION}

Every day our skin is daily aggressed by environmental factors and by certain personal care products

(cleansers, soaps, deodorants, etc). In the West, our obsession with cleanliness may do more harm than good for the balance of our skin's microbiota and skin health. Figure 1.

It has been observed that overcleanliness leads to an impairment of the skin barrier function and of skin microbiome balance leading to increased sensitivity, irritation and dryness with dysbiosis (1-2)

Although concerns have been expressed about the effect of hygiene products on the skin integrity and function, very few investigations have been conducted to show the effect of their component on the skin barrier function and skin microbiota composition.

\section{HYPOTHESIS}

Sodium Lauryl Sulfate (SLS) is a widely used surfactant in cleaning products and personal care products.

SLS is an anionic surfactant that denature cell membrane proteins. It is also known to induce irritation under occlusive patch application (3-4).

We aimed to conduct a study which had two objectives:

- Firstly, to explore the effect of SLS on skin microbiota abundance and diversity in parallel to skin barrier function evolution;

- Secondly, to explore the protective effect of a formulation containing purified natural rapeseed phytosterols.

\section{METHODS}

\section{CLINICAL PROTOCOL (Figure 2):}

Study 1: 23 females, 20-64 yo, having fair skin

(Phototype I to III), declaring a thin, dry and sensitive skin on the inner side of the forearms and showing a similar response to SLS on both forearms

- Occlusive patch containing $1 \%$ SLS for $24 \mathrm{~h}$ on both inner sides of the forearms (from Day-2 to Day-1).

Study 2: 29 healthy females, $18-45$ yo, (Phototype II \& III) with body mass index between 18.5 to $29.9 \mathrm{~kg} / \mathrm{m}^{2}$ and declaring a reactive skin

- Occlusive patch containing $0.5 \%$ SLS for $24 \mathrm{~h}$ on the upper back (from Day-2 to Day-1).

- Formulation containing 1\% purified natural rapeseed phytosterols or the vehicle of phytosterols (Cetearyl Alcohol) applied at D0, 24h after patch removal, twice a day for 7 days.

Skin barrier evaluation:

- Trans Epidermal Water Loss (TEWL) by Tewameter

- Hydration by Corneometer

Microbiota evaluation:

- Skin microbiota sampling by swabbing

- 16S rDNA gene sequencing

- Determination of skin microbiota composition and abundance

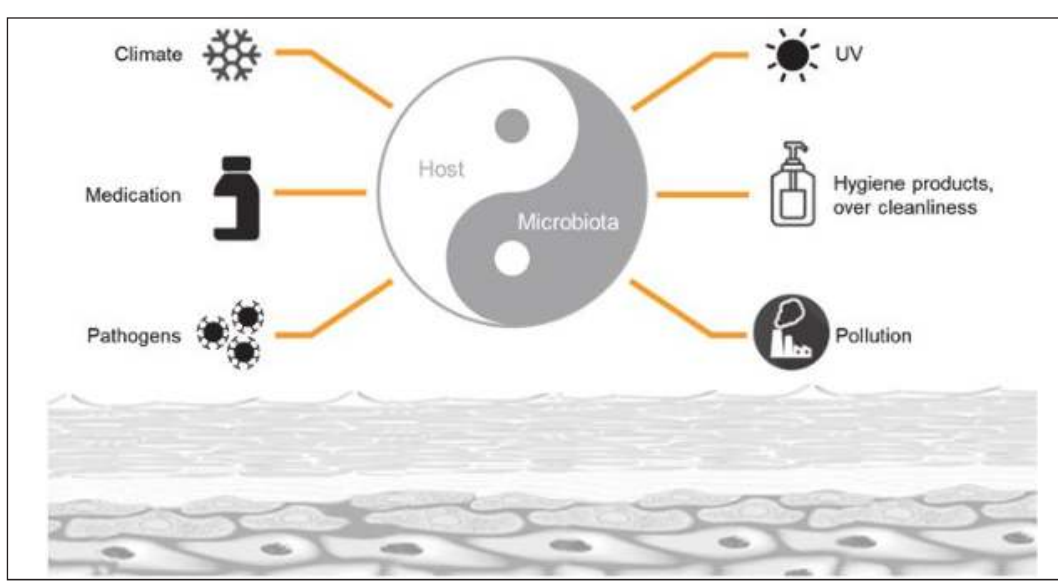

Figure 1: Beyond the physical skin barrier, the microbiotic skin barrier.

\section{RESULTS}

Physical barrier damage and restoration TEWL after $1 \%$ SLS damage

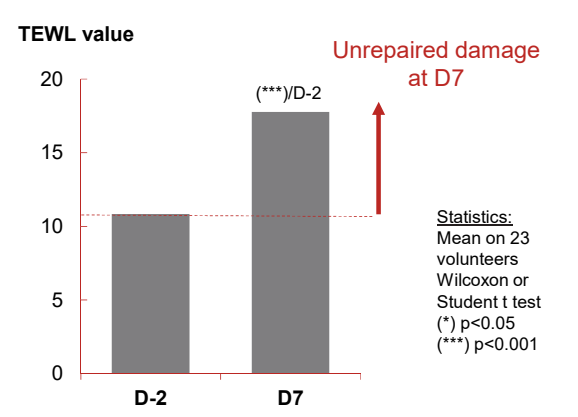
Barrier repair with $1 \%$

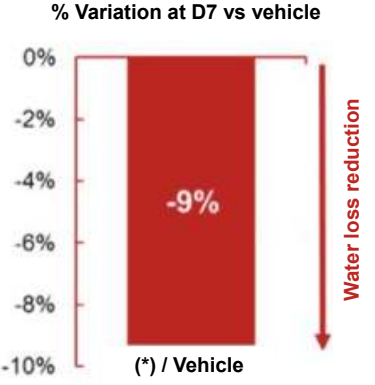

Hydration after $1 \%$ SLS damage
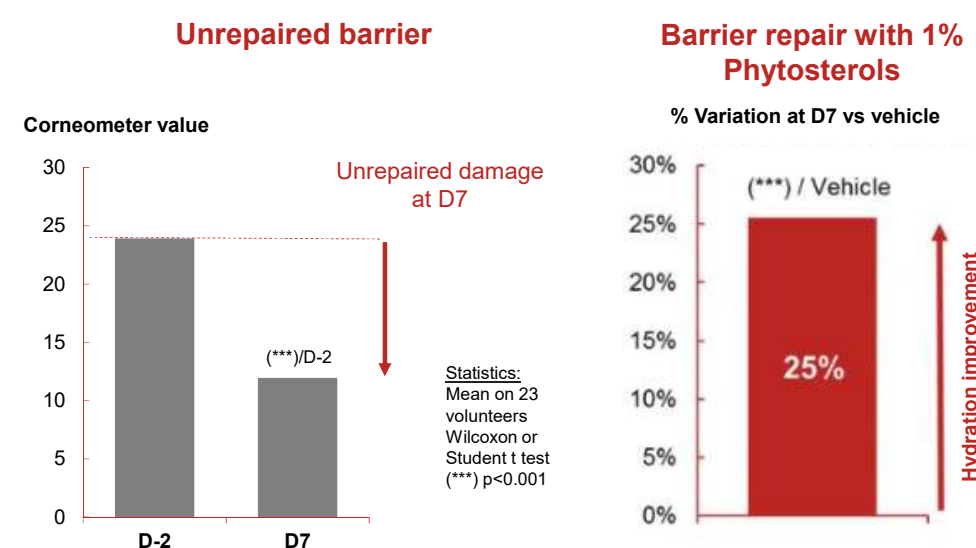

At D7, SLS skin barrier damage is unrepaired

Rapeseed Phytosterols significantly improved the recovery of the skin barrier function after impairment with SLS

\section{Microbiota ecology after $0.5 \%$ SLS damage}

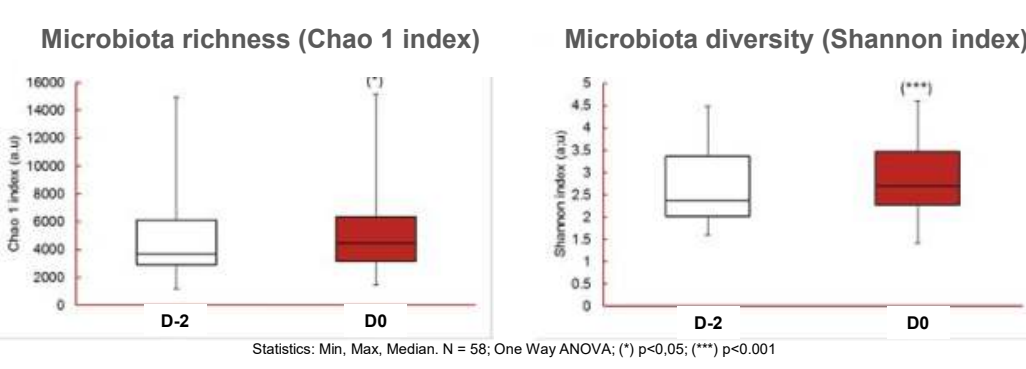

After SLS application, the number of bacterial species and the diversity increased

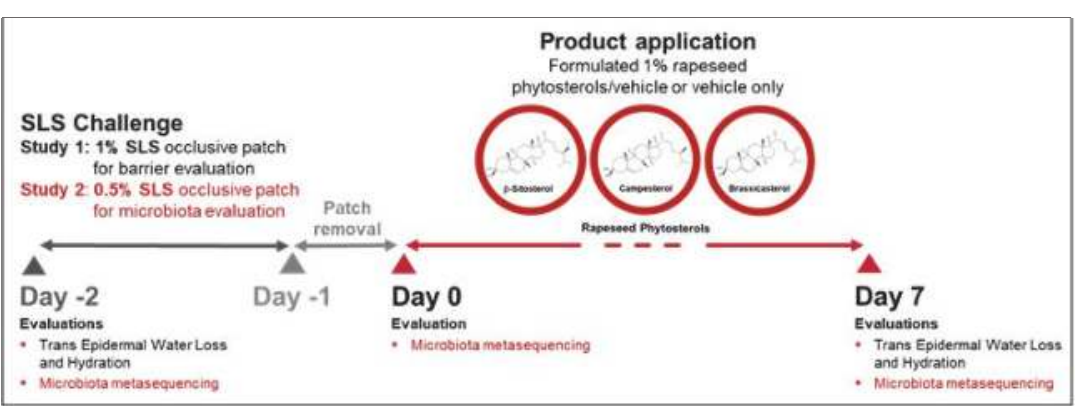

Figure 2: Clinical protocol design.

\section{Microbiota damage and restoration}

Skin microbiota before (D-2) and $24 \mathrm{~h}$ after $0.5 \%$ SLS-occlusive patch removal (DO)
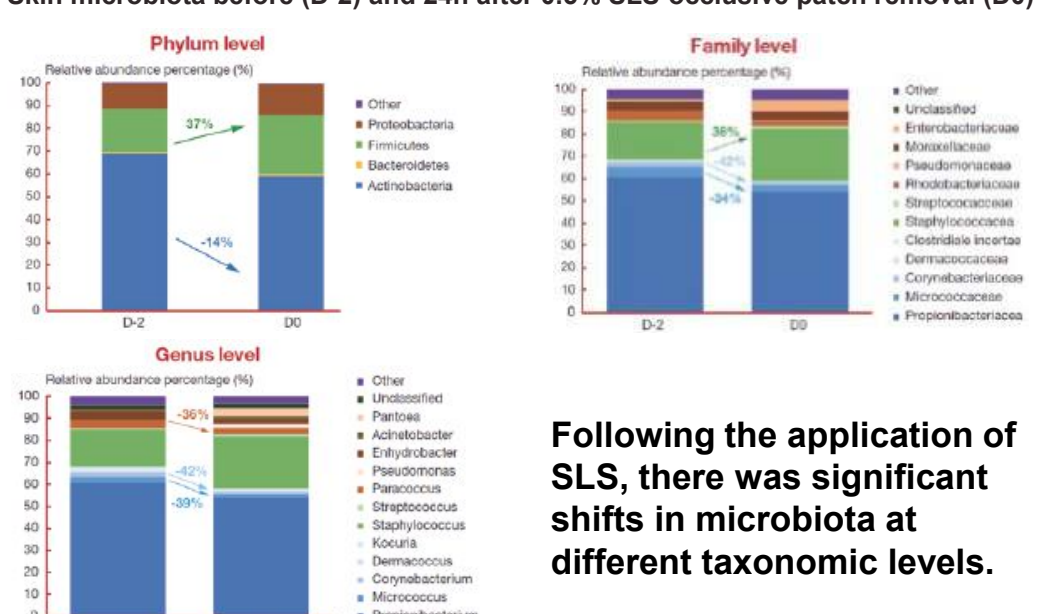

Following the application of SLS, there was significant shifts in microbiota at different taxonomic levels.

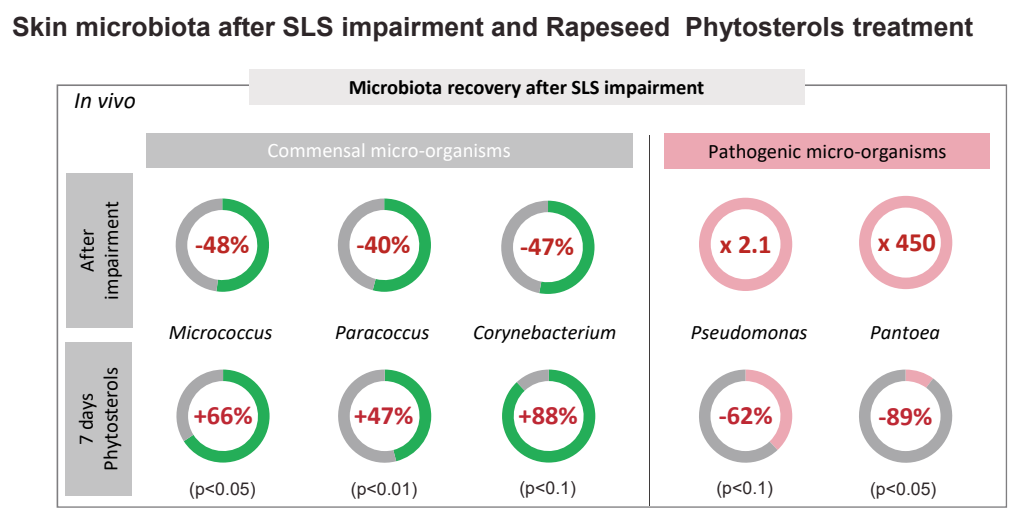

Compared to SLS induced changes, Rapeseed Phytosterols: - Increased the relative abundance of Micrococcus, Paracoccus and Reduced the relative abunc

\section{CONCLUSIONS}

Besides disrupting the physical barrier of the skin, SLS also alters the microbiotic barrier by disrupting the balance of the skin microbiota: some resident commensal microorganisms belonging to Actinobacteria (Micrococcus, Corynebacterium) and pathogenic and environmental microorganisms overgrew

The Rapeseed Phytosterols treatment helps actively to recover a beneficial resident microflora (Micrococcus, Corynebacterium and (Pantoe and Pseudomonas) for a restored microbiotic barrier.

\section{REFERENCES}

1) Rocha L.A. et al. Changes in hand microbiota associated with skin damage because of hand 2) Bojar RA, Holland $\mathrm{K}$. Review: the human cutaneous microflora and factor controlling colonization. World J. Microbiol. Biotechnol. 18: 889--903, (2002). Gabard Bet al. Surractant irititation: in vitro
Research and Technology $7: 49-55$, (2001).

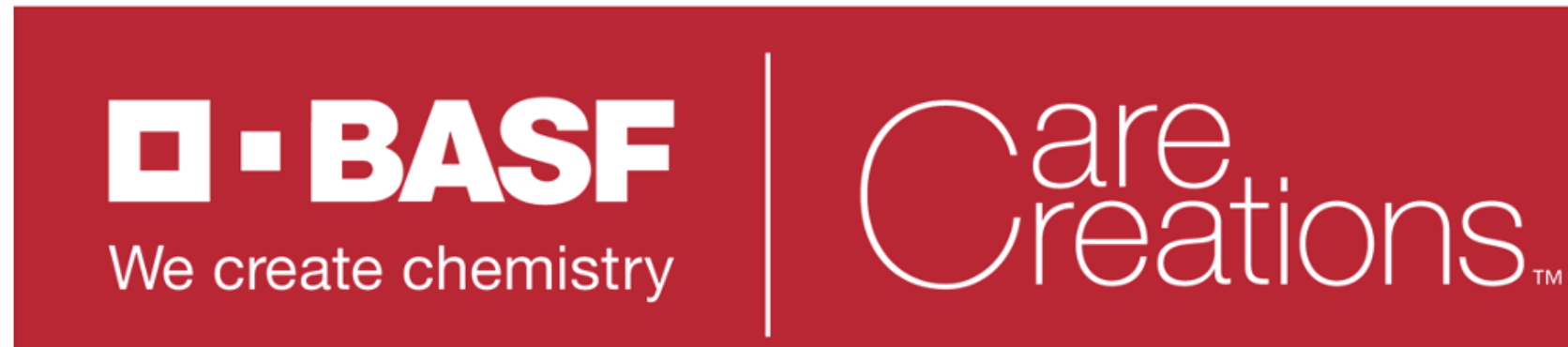

